\section{Acknowledgments}

F. Kirchhoff thanks the Wilhelm-Sander Foundation, the Deutsche Forschungsgemeinschaft, and the NIH (R01AI067057) for support.

Address correspondence to: Frank Kirchhoff, Institute of Virology, University of Ulm, Albert-Einstein-Allee 11, 89081 Ulm, Germany. Phone: 49-731-50065109; Fax: 49-731-50065131; E-mail: frank.kirchhoff@uniklinik-ulm.de. Or to: Guido Silvestri, University of Pennsylvania School of Medicine, 705 Stellar-Chance Laboratories, 422 Curie Boulevard, Philadelphia, Pennsylvania 19143, USA. Phone: (215) 5735363; Fax: (215) 573-5366; E-mail: gsilvest@ mail.med.upenn.edu.

1. Sodora, D.L., and Silvestri, G. 2008. Immune activation and AIDS pathogenesis. AIDS. 22:439-446.

2. Moses, A., Nelson, J., and Bagby, G.C., Jr. 1998. The influence of human immunodeficiency virus-1 on hematopoiesis. Blood. 91:1479-1495.

3. Marandin, A., et al. 1996. Loss of primitive hematopoietic progenitors in patients with human immunodeficiency virus infection. Blood. 88:4568-4578.

4. Sloand, E.M., et al. 1997. Secondary colony formation after long-term bone marrow culture using peripheral blood and bone marrow of HIV-infected patients. AIDS. 11:1547-1553

5. Isgrò, A., et al. 2000. Recovery of hematopoietic activity in bone marrow from human immunodeficiency virus type 1 -infected patients during highly active antiretroviral therapy. AIDS Res. Hum. Retroviruses. 16:1471-1479.
6. Baillou, C., et al. 2003. Highly active antiretroviral therapy corrects hematopoiesis in HIV-1 infected patients: interest for peripheral blood stem cellbased gene therapy. AIDS. 17:563-574.

7. Koka, P.S., Jamieson, B.D., Brooks, D.G., and Zack, J.A. 1999. Human immunodeficiency virus type 1induced hematopoietic inhibition is independent of productive infection of progenitor cells in vivo. J. Virol. 73:9089-9097.

8. Weichold, F.F., et al. 1998. Neither human immunodeficiency virus-1 (HIV-1) nor HIV-2 infects most-primitive human hematopoietic stem cells as assessed in long-term bone marrow cultures. Blood. 91:907-915.

9. Zhang, J., Scadden, D.T., and Crumpacker, C.S 2007. Primitive hematopoietic cells resist HIV-1 infection via p21. J. Clin. Invest. 117:473-481.

10. Calenda, V., Graber, P., Delamarter,J.F., and Chermann, J.C. 1994. Involvement of HIV nef protein in abnormal hematopoiesis in AIDS: in vitro study on bone marrow progenitor cells. Eur. J. Haematol. 52:103-107.

11. Reinhold, D., Wrenger, S., Kahne, T., and Ansorge, S. 1999. HIV-1 Tat: immunosuppression via TGFbeta1 induction. Immunol. Today . 20:384-385.

12. Sugiura, K., Oyaizu, N., Pahwa, R., Kalyanaraman, V.S., and Pahwa, S. 1992. Effect of human immunodeficiency virus-1 envelope glycoprotein on in vitro hematopoiesis of umbilical cord blood. Blood. 80:1463-1469.

13. Maciejewski, J.P., Weichold, F.F., and Young, N.S. 1994. HIV-1 suppression of hematopoiesis in vitro mediated by envelope glycoprotein and TNF-alpha. J. Immunol. 153:4303-4310.

14. Prost, S., et al. 2008. Human and simian immunodeficiency viruses deregulate early hematopoiesis through a Nef/PPAR $\gamma /$ STAT5 signaling pathway in macaques. J. Clin. Invest. 118:1765-1775.

15. Bunting, K.D. 2007. STAT5 signaling in nor$\mathrm{mal}$ and pathologic hematopoiesis. Front. Biosci. 12:2807-2820.

16. Socolovsky, M., Fallon, A.E., Wang, S., Brugnara, C., and Lodish, H.F. 1999. Fetal anemia and apoptosis of

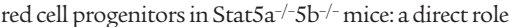

for Stat5 in Bcl-X(L) induction. Cell. 98:181-191.

17. Cui, Y., et al. 2004. Inactivation of Stat5 in mouse mammary epithelium during pregnancy reveals distinct functions in cell proliferation, survival, and differentiation. Mol. Cell. Biol. 24:8037-8047.

18. Foster, J.L., and Garcia, J.V. 2007. Role of Nef in HIV-1 replication and pathogenesis. Adv. Pharmacol. 55:389-409.

19. Schindler, M., et al. 2006. Nef-mediated suppression of $\mathrm{T}$ cell activation was lost in a lentiviral lineage that gave rise to HIV-1. Cell. 125:1055-1067.

20. Renkema, G.H., and Saksela, K. 2000. Interactions of HIV-1 NEF with cellular signal transducing proteins. Front. Biosci. 5:D268-D283.

21. Fujii, Y., Otake, K., Tashiro, M., and Adachi, A. 1996. Soluble Nef antigen of HIV-1 is cytotoxic for human CD4+ T cells. FEBS Lett. 393:93-96.

22. Federico, M., et al. 2001. HIV-1 Nef activates STAT1 in human monocytes/macrophages through the release of soluble factors. Blood. 98:2752-2761.

23. Okada, H., Takei, R., and Tashiro, M. 1997. HIV-1 Nef protein-induced apoptotic cytolysis of a broad spectrum of uninfected human blood cells independently of CD95(Fas). FEBS Lett. 414:603-606.

24. Qiao, X., et al. 2006. Human immunodeficiency virus 1 Nef suppresses CD40-dependent immunoglobulin class switching in bystander B cells. Nat. Immunol. 7:302-310.

25. Cheingsong-Popov, R. 1990. Antibodies to HIV-1 nef (p27): prevalence, significant and relationship to seroconversion. AIDS Res. Hum. Retroviruses. 6:1099-1105.

26. Silvestri, G., Paiardini, M., Pandrea, I., Lederman, M.M., and Sodora, D.L. 2007. Understanding the benign nature of SIV infection in natural hosts. J. Clin. Invest. 117:3148-3154.

27. Burchill, M.A, Yang, J., Vang, K.B., and Farrar, M.A. 2007. Interleukin-2 receptor signaling in regulatory $\mathrm{T}$ cell development and homeostasis. Immunol. Lett. 114:1-8.

28. Ye, P., Kirschner, D.E., and Kourtis, A.P. 2004. The thymus during HIV disease: role in pathogenesis and in immune recovery. Curr. HIV Res. 2:177-183.

\title{
Immunosuppression in islet transplantation
}

\section{Tom Van Belle and Matthias von Herrath}

La Jolla Institute for Allergy and Immunology, La Jolla, California, USA.

\begin{abstract}
Islet transplantation can temporarily cure type 1 diabetes mellitus (T1DM) but requires simultaneous immunosuppression to avoid allograft rejection. In this issue of the JCI, Monti et al. report that immune conditioning via use of the Edmonton protocol - a treatment approach in which T1DM patients infused with pancreatic islets from multiple cadaveric donors simultaneously receive immunosuppressive drugs - results in lymphopenia that is associated with elevated serum levels of the homeostatic cytokines IL-7 and IL-15, which causes in vivo expansion of the autoreactive $\mathrm{CD8}^{+} \mathrm{T}$ cell population (see the related article beginning on page 1806). Reemergence of autoreactivity is likely the main culprit underlying long-term islet graft failure, and new strategies will need to be tested to circumvent this homeostatic expansion and recurrent autoreactivity.
\end{abstract}

Nonstandard abbreviations used: GAD, glutamic acid decarboxylase; MMF, mycophenolate mofetil; T1DM, type 1 diabetes mellitus.

Conflict of interest: The authors have declared that no conflict of interest exists.

Citation for this article: J. Clin. Invest. 118:1625-1628 (2008). doi:10.1172/JCI35639.

\section{Autoimmunity, lymphopenia, and homeostasis}

Autoimmunity develops when immune cells attack and destroy our own cells. For instance, type 1 diabetes mellitus (T1DM) is a disease caused by immune-mediated destruction of the insulin-secreting pancreatic $\beta$ cells, leading to hyperglycemia. The immediate consequences of untreated T1DM (e.g., diabetic ketoacidosis) can be fatal, and, despite insulin therapy, long-term vascular complications can significantly affect life expectancy. Autoreactive T cells specific for $\beta$ cell autoantigens are present in most individuals, but are usually kept under control by peripheral tolerance mechanisms or have a regulatory phenotype (1). It is now well established that T1DM is the result of interactions between susceptibility genes (especially those within the HLA region) and probably several environmental factors (2). The highest-risk genotypes, HLA-DR3 or HLA-DR4 class II alleles, can be responsible for the development of anti-islet autoimmunity via production of autoantibodies to insulin; to glutamic acid 


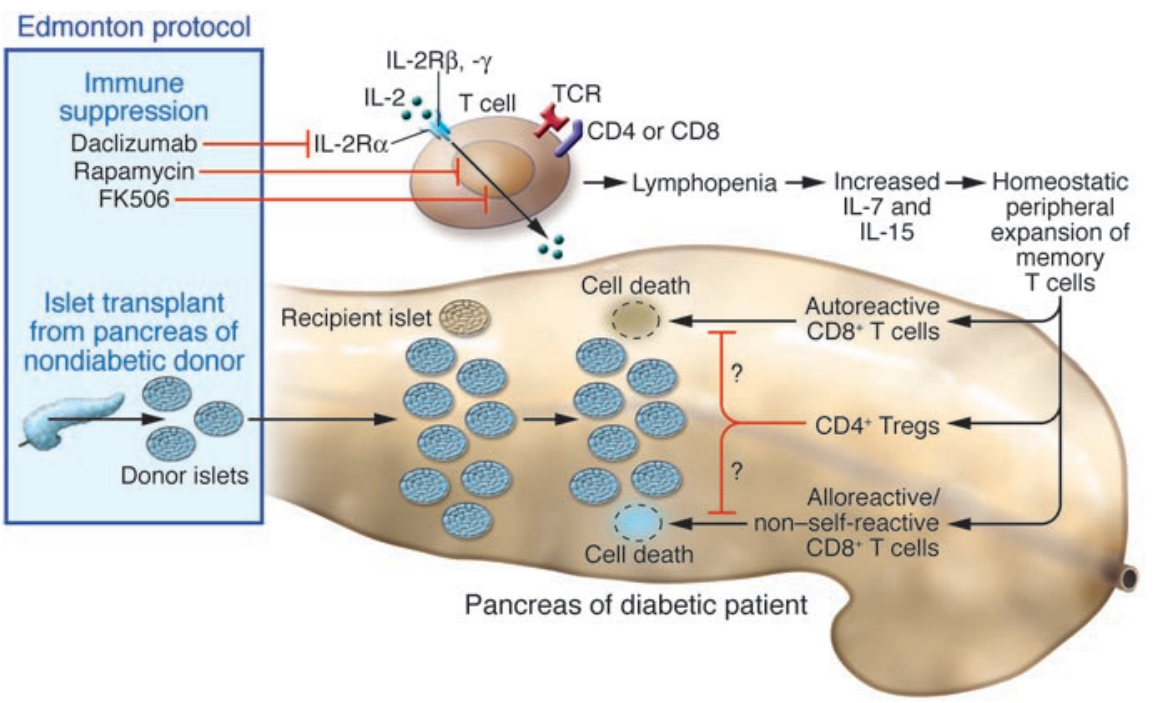

Figure 1

The immunologic consequences of islet transplantation under the Edmonton protocol. Pancreatic islets of diabetic patients can be infiltrated, destroyed, or dysfunctional, which renders them unable to produce insulin. Therefore, as a potential cure, islets from nondiabetic donors are transplanted using immunosuppressive regimens. The Edmonton protocol had shown the most promise so far, although recently it became clear that most islet grafts are lost after 5 years (5). In this issue of the $\mathrm{JCl}$, Monti et al. (6) now show that the Edmonton protocol of immunosuppression with daclizumab, rapamycin, and FK506 results in lymphopenia and thus homeostatic expansion of autoreactive CD8 ${ }^{+} \mathrm{T}$ cells. Lymphopenia elicits production of IL-7 and IL-15 that drives homeostatic peripheral expansion of memory T cells, which comprise autoreactive, alloreactive, and probably other T cell populations. In addition, the lack of IL-2 and blockade of the IL-2 signaling pathway might affect not only effector $T$ cells, but also Tregs. The reemerging autoreactive T cells are capable of destroying the patient's remaining $\beta$ cells (brown) and the donor islets (blue), which are transplanted via the hepatic portal vein. It is unknown whether enhanced homeostatic proliferation of Tregs with different immunosuppressive regimens or augmentation of islet-specific Tregs after vaccination or cell transfer could be sufficient to inhibit any of the $\beta$ cell-destructive T cell responses.

decarboxylase (GAD), an enzyme produced primarily by pancreatic islet cells; and to the transmembrane protein tyrosine kinase IA-2, which regulates vesicle number and insulin secretion. However, it is likely that an environmental event like a viral infection or lymphopenia needs to occur to precipitate disease (3).

\section{Immunosuppression and reemergence of autoimmunity}

First devised in the late 1990s at the University of Alberta, the Edmonton protocol outlines a method of transplanting pancreatic islets from multiple cadaveric donors into individuals with T1DM and subsequent administration of immunosuppressants and a $\mathrm{mAb}$, in an effort to restore insulin independence or at least sufficient islet function to protect against hypoglycemia (Figure 1) (4). Specifically, immunosuppression begins immediately before transplantation, with 5 biweekly doses of a humanized anti-IL-2 receptor $\alpha$ (anti-IL-2R $\alpha$ ) mAb known as daclizumab (Zenapax; Roche), and is main- nosuppression is associated with elevated serum concentrations of the cytokines IL-7 and IL-15 and an increased population of proliferating lymphocytes positive for the cell proliferation-associated antigen Ki-67. These Ki- $67^{+}$cells are unable to produce the multifunctional effector cytokine IFN- $\gamma$, which is known to foster inflammation and islet destruction in T1DM. However, switching the immunosuppressive regimen in 2 patients from rapamycin and FK506 - because of rapamycin intolerability - to mycophenolate mofetil (MMF, CellCept; Roche), which selectively inhibits $\mathrm{T}$ and $\mathrm{B}$ cell proliferation by suppressing de novo purine synthesis, blocked lymphocyte proliferation despite sustained high levels of IL-7 in serum. Data on the in vivo effector $\mathrm{T}$ cell function or the clinical outcome in these 2 patients were not reported. In vitro cultures of the lymphocytes from patients on the Edmonton protocol proliferated vigorously without overt stimulation. Interestingly, this spontaneous proliferation was blocked by MMF, but not by rapamycin, FK506, or cyclosporin A. Effector functions that were absent in vivo became obvious after 48 hours of culture, as measured by IFN- $\gamma$ production, indicative of an expanding effector memory $\mathrm{T}$ cell population. In these expanded in vitro cultures, inhibition of IFN- $\gamma$ production was better achieved with rapamycin, but not with MMF. Thus, MMF can block proliferation and expansion of PBMCs as well, whereas rapamycin is better at curbing IFN- $\gamma$ production.

In the current study (6), pentamer staining showed increased numbers of not only influenza-specific T cells, but, more importantly, GAD65-autoreactive T cells, 30\% of which proliferated in vivo and expressed the CD45RO (memory) marker. This homeostatic expansion of autoreactive $T$ cells may well explain the limited success of the Edmonton protocol over time. Indeed, the population of preexisting GAD65-autoreactive memory T cells could be expanded by peptide stimulation in vitro from the memory $\mathrm{CD}^{+} \mathrm{T}$ cell pool, which was further enhanced by IL-7. In this context, a very interesting report by Roep and colleagues is noteworthy: these authors showed that patients with preexisting cellular autoimmunity to GAD lost islet graft function more rapidly than did patients without preexisting cellular autoimmunity to GAD, despite immunosuppression with the Edmonton protocol (7).

One previous study in NOD mice directly linked T1DM autoimmunity with lymphopenia and increased IL-21 production (8). 
Recently, experimentally induced lymphopenia was shown to increase diabetes onset dramatically in a CD4 ${ }^{+} \mathrm{T}$ cell transfer model (9). Direct support for an association of human T1DM with lymphopenia or IL-21 serum levels has been lacking, but the genetic murine Idd3 and human 4q26-q27 intervals encoding IL-21 and IL-2 convey susceptibility to T1DM in both mice and humans (10). Many viral infections, such as measles and SARS $(11,12)$, can cause transient lymphopenia, and IL-21 is upregulated in vivo upon infection (13). In this context, it is important to note that infections with enteroviruses (notably coxsackievirus B4; ref. 14) or rotaviruses (15) have been associated with the emergence of islet autoantibodies or even development of clinical T1DM. Lymphopenia triggers homeostatic mechanisms in our immune system (16), which involve MHC contact for naive T cells, but for only a minor fraction of memory $\mathrm{T}$ cells. Multiple cytokines play an important role in $\mathrm{T}$ cell homeostasis (17), and under normal physiological conditions, homeostatic proliferation of $\mathrm{CD}^{+}$and $\mathrm{CD}^{+}$memory cells is driven by IL-15 and IL-7 (18). In lymphopenic hosts, however, memory $\mathrm{CD}^{+} \mathrm{T}$ cells ignore IL-15 and expand in response to the elevated IL-7 levels, whereas memory CD8 ${ }^{+}$ $\mathrm{T}$ cells have a strong bias for IL-15 over IL-7 (16). These observations are relevant to the present report by Monti et al. (6), because elevated levels of both IL-7 and IL-15 were found during immune conditioning using the Edmonton protocol.

\section{Tregs and homeostasis}

Human $\mathrm{CD}^{+} \mathrm{T}$ cells include a subset of CD $4^{+} \mathrm{CD} 25^{\text {hi }}$ Tregs that can efficiently suppress effector $\mathrm{T}$ cell responses. While the absolute numbers of Tregs are clearly diminished in lymphopenic mice, Tregs themselves can be homeostatically expanded (3). In fact, humans rendered lymphopenic by cyclophosphamide display a relative increase in the frequency of Tregs compared with healthy individuals (19). This suggests that Treg expansion itself is an important safeguard to limit autoimmune reactivity during lymphopenia (3). Therefore, data on the loss or homeostatic expansion of Tregs following systemic immunosuppression with the Edmonton protocol would be very interesting. Because Tregs depend on IL-2 for proliferation $(20,21)$, a blockade of IL-2 signaling and production during immunosuppression by the Edmonton protocol may lower proliferation of not only effector cells, but also Tregs.

\section{Future directions: choosing the best} immunosuppressive regimen

The strength of the present report is the demonstration that homeostatic mechanisms can lead to the amplification of autoreactive memory $T$ cells during interventions that are targeted toward the opposite outcome, i.e., curing autoimmunity. This could be specific to the Edmonton protocol and is not necessarily inherent to all immunosuppressive protocols. An ideal immunosuppressive therapy would therefore minimize lymphodepletion and preserve Treg function, maybe by avoiding targeting cytokines that are required for Treg expansion and function or by directly inducing Tregs specific for islet antigens. For example, an ongoing phase II clinical trial addresses the therapeutic potential of MMF and daclizumab combination therapy in recent-onset T1DM patients without islet transplantation (22). Promising results have been achieved by administering humanized nonmitogenic and nondepleting anti-CD3 mAbs to individuals with T1DM, resulting in preservation of C-peptide levels for up to 2 years (23), and recent animal studies show that anti-CD3 can synergize with islet antigen-specific approaches to induce tolerance via Treg induction (24). A phase III clinical trial will investigate the efficacy of other immunosuppressive drugs (antithymocyte globulin, daclizumab, etanercept, sirolimus, or tacrolimus) in combination with islet transplantation (25). It will be interesting to see whether homeostatic issues emerge.

Overall, the reported expansion of autoreactive $T$ cells provides a possible explanation for the limited long-term success of the Edmonton protocol in T1DM islet transplantation. Importantly, a recent study showed that the immunosuppressive agents used in the Edmonton protocol can also block $\beta$ cell regeneration (26), putting yet another burden on refining such interventions further. Future clinical trials should monitor the appearance of homeostatic cytokines and proliferating autoreactive memory $\mathrm{T}$ cells, Tregs and their function, and $\beta$ cell mass, if possible. In addition, the numbers of autoreactive effector $T$ cells producing IFN- $\gamma$ prior to transplantation should be used as a prognostic tool (7). We are just beginning to amend immunosuppressive therapy in T1DM to cope with preexistent immunity against the graft, while most drugs currently used in islet transplantation are primarily geared to prevent allograft rejection. The recent successes achieved in mice with antigen-specific induction of Tregs by administration of nasal proinsulin (24), a proinsulin-based DNA vaccine (27), or the GAD65 protein offered by Diamyd Medical (28) support the notion that antigen-specific immunotherapy should form a substantial part of future refinement of immunosuppressive regimens. Antigen-specific induction of Tregs could warrant long-term maintenance of tolerance without continuous immunosuppression.

\section{Acknowledgments}

T. Van Belle is supported by a fellowship from the Belgian American Exchange Foundation (BAEF). The authors acknowledge $S$. Lagaert for help with creating Figure 1.

Address correspondence to: Matthias von Herrath, La Jolla Institute for Allergy and Immunology, 9420 Athena Circle, La Jolla, California 92037, USA. Phone: (858) 752-6817; Fax: (858) 752-6993; E-mail: matthias@liai.org.

1. Bach, J.F., and Chatenoud, L. 2001. Tolerance to islet autoantigens in type 1 diabetes. Annu. Rev. Immunol. 19:131-161.

2. Gianani, R., and Eisenbarth, G.S. 2005. The stages of type 1A diabetes: 2005. Immunol. Rev. 204:232-249.

3. Krupica, T., Jr., Fry, T.J., and Mackall, C.L. 2006. Autoimmunity during lymphopenia: a two-hit model. Clin. Immunol. 120:121-128.

4. Shapiro, A.M., et al. 2000. Islet transplantation in seven patients with type 1 diabetes mellitus using a glucocorticoid-free immunosuppressive regimen. N. Engl. J. Med. 343:230-238.

5. Shapiro, A.M., et al. 2006. International trial of the Edmonton protocol for islet transplantation. N. Engl. J. Med. 355:1318-1330.

6. Monti, P., et al. 2008. Islet transplantation in patients with autoimmune diabetes induces homeostatic cytokines that expand autoreactive memory T cells. J. Clin. Invest. 118:1806-1814.

7. Huurman, V.A.L., et al. 2008. Cellular islet autoimmunity associates with clinical outcome of islet cell transplantation. PLOS ONE. In press.

8. King, C., Ilic, A., Koelsch, K., and Sarvetnick, N. 2004. Homeostatic expansion of T cells during immune insufficiency generates autoimmunity. Cell. 117:265-277.

9. Calzascia, T., et al. 2008. CD4 T cells, lymphopenia, and IL-7 in a multistep pathway to autoimmunity. Proc. Natl. Acad. Sci. U. S. A. 105:2999-3004.

10. Wicker, L.S., et al. 1994. Resistance alleles at two non-major histocompatibility complex-linked insulin-dependent diabetes loci on chromosome 3 , Idd 3 and Idd 10 , protect nonobese diabetic mice from diabetes. J. Exp. Med. 180:1705-1713.

11. Wesley, A., Coovadia, H.M., and Henderson, L. 1978. Immunological recovery after measles. Clin. Exp. Immunol. 32:540-544.

12. Lau, Y.L., and Peiris, J.S. 2005. Pathogenesis of severe acute respiratory syndrome. Curr. Opin. Immunol. 17:404-410.

13. Holm, C., Nyvold, C.G., Paludan, S.R., Thomsen, A.R., and Hokland, M. 2006. Interleukin21 mRNA expression during virus infections. Cytokine. 33:41-45.

14. Marttila, J., Hyoty, H., Nanto-Salonen, K., Simell, O., and Ilonen, J. 2004. Epitopes recognized by CBV4 responding T cells: effect of type 1 diabetes and associated HLA-DR-DQ haplotypes. Virology. 319:27-35.

15. Ballotti, S., and de Martino, M. 2007. Rotavirus 
infections and development of type 1 diabetes: an evasive conundrum. J. Pediatr. Gastroenterol. Nutr. 45:147-156

16. Surh, C.D., Boyman, O., Purton, J.F., and Sprent, J. 2006. Homeostasis of memory T cells. Immunol. Rev. 211:154-163.

17. Dooms, H., and Abbas, A.K. 2006. Control of CD4+ T-cell memory by cytokines and costimulators. Immunol. Rev. 211:23-38.

18. Purton, J.F., et al. 2007. Antiviral CD4+ memory T cells are IL-15 dependent. J. Exp. Med. 204:951-961.

19. de Kleer, I., et al. 2006. Autologous stem cell transplantation for autoimmunity induces immunologic self-tolerance by reprogramming autoreactive $\mathrm{T}$ cells and restoring the CD4+CD25+ immune regulatory network. Blood. 107:1696-1702.
20. Malek, T.R., and Bayer, A.L. 2004. Tolerance, not immunity, crucially depends on IL-2. Nat. Rev. Immunol. 4:665-674.

21. Boyman, O., Kovar, M., Rubinstein, M.P., Surh, C.D., and Sprent, J. 2006. Selective stimulation of $\mathrm{T}$ cell subsets with antibody-cytokine immune complexes. Science. 311:1924-1927.

22. NIDDK. New onset of type 1 diabetes mycophenolate mofetil-daclizumab clinical trial [ClinicalTrials. gov study NCT00100178]. http://www.clinicaltrials. gov/ct2/show/NCT00100178.

23. Keymeulen, B., et al. 2005. Insulin needs after CD3antibody therapy in new-onset type 1 diabetes. N. Engl. J. Med. 352:2598-2608.

24. Bresson, D., et al. 2006. Anti-CD3 and nasal proinsulin combination therapy enhances remission from recent-onset autoimmune diabetes by inducing Tregs. J. Clin. Invest. 116:1371-1381.

25. NIAID. Islet transplantation in type 1 diabetes [ClinicalTrials.gov study NCT00434811]. http:// www.clinicaltrials.gov/ct2/show/NCT00434811.

26. Nir, T., Melton, D.A., and Dor, Y. 2007. Recovery from diabetes in mice by beta cell regeneration. J. Clin. Invest. 117:2553-2561.

27. Bayhill Therapeutics. Phase 1 study of BHT-3021 in subjects with type 1 diabetes mellitus [ClinicalTrials.gov study NCT00453375]. http://www.clinicaltrials.gov/ct2/show/NCT00453375.

28. NIDDK. Effects of recombinant human glutamic acid decarboxylase [ClinicalTrials.gov study NCT00529399]. http://www.clinicaltrials.gov/ct2/ show/NCT00529399.

\title{
What lurks beneath: IL-11, via Stat3, promotes inflammation-associated gastric tumorigenesis
}

\author{
Juanita L. Merchant \\ Department of Internal Medicine and Department of Molecular and Integrative Physiology, \\ Division of Gastroenterology, University of Michigan, Ann Arbor, Michigan, USA.
}

\begin{abstract}
Chronic inflammation in the stomach induces cellular transformation and gastric cancer primarily in the distal stomach or antrum. In this issue of the JCI, a study in mice by Ernst et al. provides new insight into the role of IL-11 and its glycoprotein 130 (gp130) receptor in inflammation-associated gastric epithelial cell oncogenic transformation, which they show is mediated by and dependent on increased activation of Stat 3 and, to a lesser extent, Stat1 (see the related article beginning on page 1727). Prior studies from this group have shown that Stat 3 hyperactivity stimulates the TGF- $\beta$ inhibitor Smad7. Collectively, the studies suggest that an important pathway of oncogenic transformation in the stomach is through suppression of growth inhibitory signals, such as members of the TGF- $\beta$ family, that originate from the stroma.
\end{abstract}

Cancer originates in a variety of different tissues that are susceptible to chronic inflammatory conditions. In the stomach, the phenotypic steps leading from chronic inflammation (gastritis) to distal gastric cancer were established epidemiologically (1) before the discovery of Helicobacter pylori, a noninvasive curvilinear rod that resides in the luminal mucous layer (2). Although H. pylori appears to be the most common infectious agent inducing chronic gastritis, studies support the notion that inflammatory cells infiltrate the mucosa from the stroma beneath and secrete cytokines that are ostensibly the direct modulators of epithelial cell differentiation and eventually neoplastic transformation $(3,4)$. If $H$. pylori is not eradi-

Nonstandard abbreviations used: gp130, glycoprotein 130; Smad7, mothers against decapentaplegic homolog 7 .

Conflict of interest: The author has declared that no conflict of interest exists.

Citation for this article: J. Clin. Invest. 118:1628-1631 (2008). doi:10.1172/JCI35344. cated by the inflammatory response, the bacterium becomes a chronic trigger for the immune system to produce cytokines that continue to bathe the gastric glands. Metaplastic changes begin in the antrum, where H. pylori preferentially colonizes the stomach, before spreading anteriorly into the corpus, inducing atrophy of the oxyntic glands (5). Eventually, mucus-producing, metaplastic cells that exhibit features of either the antral pyloric glands (via antralization) or small intestine replace the normal oxyntic (acid/ pepsin-producing) cells $(6,7)$. These specific changes in the gastric lineages launch the mucosa down an irreversible pathway toward unregulated growth, dysplasia, and eventually neoplastic transformation.

Population-based studies by El-Omar et al. indicate that IL- $1 \beta$ is one of the essential proinflammatory cytokines modulated during H. pylori infection that directs the mucosa toward atrophy, metaplasia, and neoplastic transformation (8). In European, Asian, and Hispanic populations, the presence of a gas- tric cancer-associated polymorphism has not only been confirmed for IL- $1 \beta$, but also reported for TNF- $\alpha$, IL-10, and IL-6 (9-11). Collectively, these prior studies have set in motion the quest to link inflammation and specific cytokines with cancer development. The underlying issue is that once inflammation becomes chronic, antibiotic eradication of the triggering organism would not be an effective treatment. It remains to be seen whether antiinflammatory therapies that do not disrupt mucosal restitution can be used.

\section{A fully penetrant model of distal gastric cancer}

To study the etiology of gastric transformation, a number of mouse models have been developed that recapitulate several features observed in human intestinal-type gastric cancer (Table 1). The gp1307757F/Y757F mouse studied by Ernst et al. in this issue of the JCI (12) is the mouse model that most consistently develops gastric cancer. These animals are homozygous for a phenylalanine knockin substitution at Tyr757, which is present in one of five major phosphorylation sites in the cytoplasmic tail of the glycoprotein 130 (gp130) receptor. This receptor - the common receptor for members of the IL- 6 cytokine family - is important for signal transduction following cytokine engagement. Upon binding of an IL-6 cytokine family member to its relevant receptor, the complex of these two proteins associates with gp130 and homodimerizes to form a hexameric complex that upon phosphorylation 